\title{
Metabolic Syndrome in Endocrine System
}

\author{
Vanita Pudata* and Jhansi Konduru
}

Department of Biochemistry, Dr. L. Bullayya College, Andhra University, Visakhapatnam, India

\begin{abstract}
Hormonal change in endocrine glands leads to metabolic syndrome. Latest research work suggests that nearly $40-50 \%$ of peoples only from USA suffer due to metabolic syndrome. Sedentary lifestyle leads to metabolic syndrome. Commonly seen metabolic syndromes are cardio-metabolic syndrome, syndrome X, insulin resistance syndrome, Reaven's syndrome which leads to diabetes, Obesity, pancreatic disorders, etc. Hormone like insulin, which is produced from $\beta$ - cells of pancreas plays vital role in metabolic syndrome. Not only insulin but metals (Arsenic, cadmium, copper, lead, manganese, molybdenum, iodine) and proteins also have major role in metabolic syndrome. Metabolic syndrome percentage is more in women than that of men. The patients who are suffering from HIV, they commonly have metabolic syndromes. Dietary habit and sedentary lifestyle is a one of the causes for the metabolic syndrome.
\end{abstract}

Keywords: Systemic inflammatory response syndrome; Highly active antiretroviral therapy; Type 2 diabetes mellitus; Parent-only; Obstructive sleep apnea; High levels of bad cholesterol; Low levels of good cholesterol; Cardiovascular diseases; Hormone replacement therapy

\section{Introduction}

Endocrine system produces different hormones and controls the important body function \& development, cell regulation \& metabolism. A hormone imbalance in endocrine system leads to metabolic syndromes like cardio-metabolic syndrome, syndrome $\mathrm{X}$, insulin resistance syndrome, Raven's syndrome which leads to diabetes, Obesity, (Figure 1) pancreatic disorders, etc. [1]. Metabolic syndrome is not only risk factor for diabetes but also for cardiovascular diseases [2]. Recent research studies shows that the deficiency of vitamin D causes metabolic syndrome, diabetes mellitus, heart failure, stroke, cancer, polycystic ovary syndrome, (Figure1) gout, and asthma and coronary artery disorders [3]. Osteoporosis is also frequently seen metabolic syndrome, which decreases the bone formation and number of cells in bone, increases the risk of bone fractures [4]. Metals like zinc, arsenic, cadmium, copper, lead, manganese, molybdenum and iodine also play a vital role in prevention of metabolic syndrome. Zinc not only improves insulin sensitivity in diabetes but also utilizes the glucose in absence of insulin [5]. Arsenic, cadmium, copper, lead, manganese, molybdenum and iodine associates with chromium and regulates thyroid hormone synthesis [6]. Proteins also have crucial role in prevention of metabolic syndromes as if hormonal regulation also depends upon amino acid

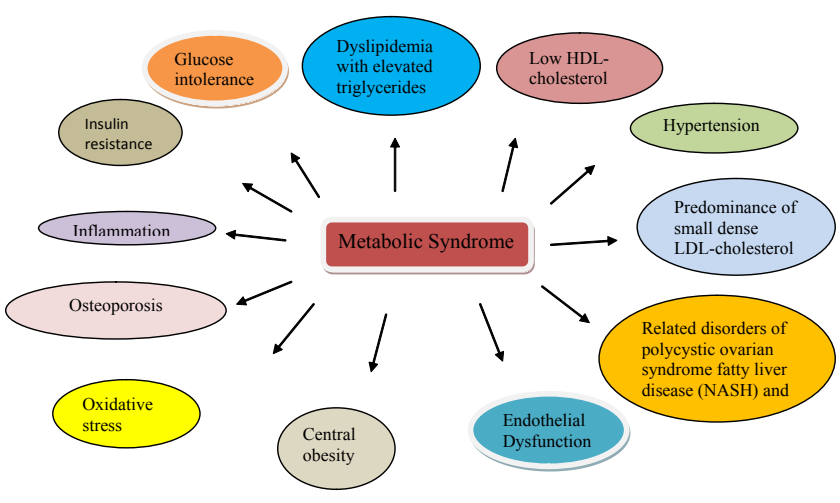

Figure 1: Related Disorders in metabolic Syndrome
[7]. Protein p21 is main regulator for apoptosis and cell differentiation in non-various tissues [8]. As we all know hormones are the one which plays major role in metabolic syndrome, it circulate through out body by blood stream. Some endocrine glands like pituitary gland, adrenal gland, thyroid gland, thymus and pancreatic gland produces hormones. The imbalance in these hormonal levels leads to metabolic syndrome [9]. Latest research studies show that the people who were living with HIV also suffers from many metabolic syndromes [10]. The metabolic syndrome in HIV patients results in the increase of blood sugar and lipid levels which leads to diabetes and have higher risk than that of the HIV uninfected persons [11]. The HIV patients those who use highly active antiretroviral therapy (HAART) have high complications of metabolic syndromes like diabetes, high blood pressure, abdominal obesity, low HDL, hypercholesterolemia, hypertriglyceridemia and cardiovascular disorders [12]. Due to alterations in the levels of $\beta$-protein one can suffer from syndromes like Down syndrome, Fragile $\mathrm{X}$ syndrome, Parkinson's disease and Alzheimer's disease [13]. Stress in cytokines in the hypothalamic-pituitary-adrenal axis leads to Systemic Inflammatory Response Syndrome (SIRS) [14]. Enzyme alteration cause reduces the metabolic rate and which leads to the disorders [15]. Bacteria named staphylococcacea is the main cause for cardiovascular infections, Scalded Skin Syndrome, Toxic Shock Syndrome and deadly diseases [16]. Enzyme alteration cause reduces the metabolic rate and which leads to the disorders [15]. Staphylococcacea is the main cause for cardiovascular infections, Scalded Skin Syndrome, Toxic Shock Syndrome and deadly diseases [16]. Metabolic syndrome is also marked as hypertension, complication in nephropathy, retinopathy and neuropathy [17]. Hyperinsulinemia is also one of the major metabolic syndromes of type 2 diabetes [18].

Metabolic Syndrome also affects all age groups. Latest studies shows; in U.S.A nearly $44 \%$ of above 50 aged people are suffering

*Corresponding authors: Vanita Pudata, Department of Biochemistry, Dr. L. Bullayya college, Andhra University, Visakhapatnam, India, E-mail: vani.vnt1@ gmail.com

Received November 24, 2011; Accepted November 30, 2011; Published December 02, 2011

Citation: Vanita P Jhansi K (2011) Metabolic Syndrome in Endocrine System. Diabetes Metab 2:163. doi:10.4172/2155-6156.1000163

Copyright: (C) 2011 Vanita P, et al. This is an open-access article distributed unde the terms of the Creative Commons Attribution License, which permits unrestricted use, distribution, and reproduction in any medium, provided the original author and source are credited. 
from metabolic syndrome. Not only age but stress is also one of the sources for metabolic syndromes. Sedentary lifestyle also leads to metabolic syndrome [19]. Though maximum number of persons are suffering from metabolic syndrome there is a solution to prevent this by changing their lifestyle. New techniques also developed for the treatment of metabolic syndrome like microcirculation. One of the processes of microcirculation is microvascular vasodilatation which is used to demonstrate diabetes and metabolic syndrome but complete pathogenicity of insulin resistance cannot be determining [20]. Nutrition also plays an important role to prevent metabolic syndrome, intake of poor diet of an individual can also cause different metabolic syndrome [21].

\section{Metabolic Syndrome}

Now a day's life style became very adventives so that every work became easy to do which leads to different disease so called metabolic syndrome. Diabetes, obesity, cardiovascular diseases, hormonal imbalance are some of the metabolic syndrome [22].

Diabetes: Diabetes is an enduring disease which can't be cured but can be prevented by taking care. It is one of the commonly found metabolic syndromes everywhere in the world. Diabetes mellitus mainly associates with central nervous system and peripheral nervous system which are long term complications [23]. According to the 2006 WHO survey, nearly $10.4 \%$ people suffering from diabetes in Iraq [24]. In Asian countries (Figure 2) such as India and China there is an increased factor of diabetes [25]. Worldwide the percentage of diabetes is more in women and men i.e. male have 47 from 1000 and 58 female from 1000 members [26].Alterations in the blood glucose level leads to diabetes. Diabetes mellitus is a commonly seen metabolic syndrome of diabetes which shares the phenotype of hyperglycemia [27]. The diabetic patients with osteoporosis cause increased osteoporotic fractures [28]. It is a chronic disease; the body cannot use glucose and stores in blood which damage kidneys, nerves, heart, eyes, and blood vessels [29].

There are three types of diabetes risk factors:

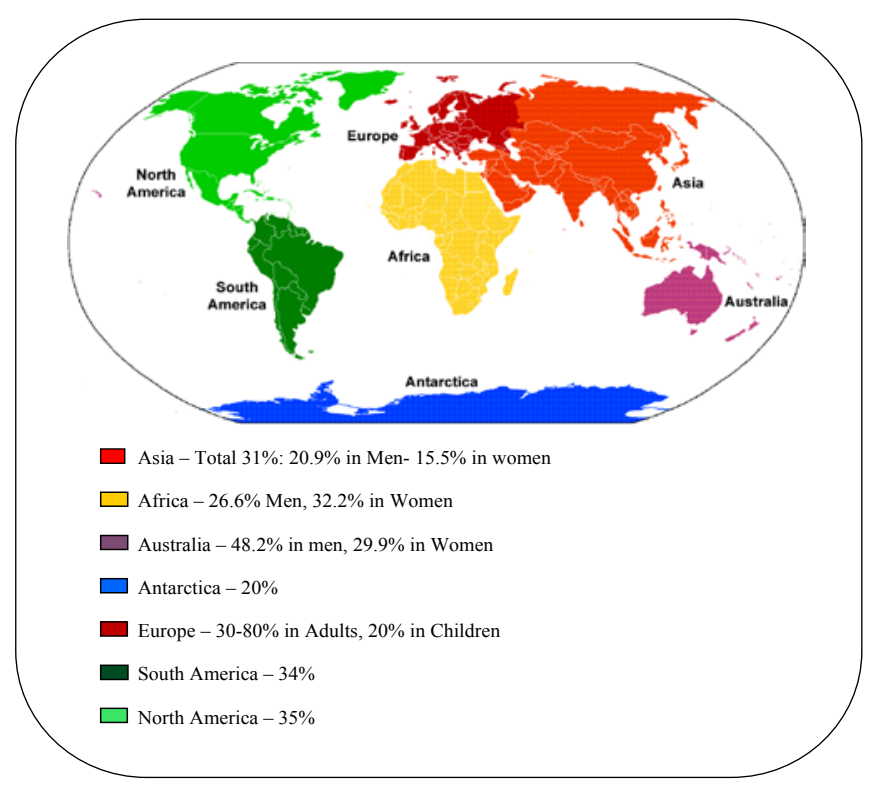

Figure 2: Different percentage of metabolic syndrome in continents

\section{1) Type1 Diabetes}

2) Type 2 Diabetes

3) Gestational Diabetes

Type 1 and type 2 diabetes may occur in any age group. The people who are suffering from type 1 diabetes need insulin injection daily where as type 2 diabetic patients does not require daily insulin injections. Gestational diabetes is the condition in pregnant women where the blood sugar increases thought may not have diabetes [30]. In some cases it lasts even after the delivery. Women with gestational diabetes mellitus (GDM) history also have increased risk of type 2 diabetes and cardiovascular diseases [31]. Insulin antagonism in type 2 diabetes leads to polygenic abnormalities like cleft lip, cleft palate, neural tube defect. The genetic factor, age, weight also influence high risk of diabetes. Diabetes can cause high risk of nocturnal hypoglycemia which is severe in children leads brain damage [32].

Cause: Metabolic Syndrome like diabetes occurs due to insulin imbalance, which is synthesized by $\beta$-cells of the pancreas. The $\beta$-cells of the pancreas are produced from Ilets of Langerhans. Insulin plays a key role in glucose uptake and utilization [33]. Dysfunction of $\beta$-cells results in deficiency of both insulin and amylin which increases weight in diabetic patients [34]. The hepatocyte nuclear factor 4- $\alpha$ (HNF4A) is responsible for the gene transcription of pancreatic $\beta$-cells here hepatic gene plays an imperative role. In type 2 diabetes has heterogenetic, and non-ketone sickness tendency and about $2 \%-5 \% \beta$ cell dysfunction seen in type 2 diabetic people [35]. Though the mutation in hepatic gene leads to maturity-onset diabetes of the young ones, it is non-insulindependent diabetes [36]. Gestational diabetes mellitus occurs mainly during pregnancy and causes risk to both mother and neonate [37]. Diabetes can also be treated by the intake of drugs, which contains a-glycosidase inhibitors and also can be treated by maintaining proper diet and exercise [38]. Bar-reflex is a new mechanism which enhances morbidity and mortality in diabetic patients by changing the heart beat [39]. Insulin resistance also disturbs the lipid metabolism and increase fatty acid leads to obesity, type 2 diabetes and cardiovascular diseases [40]. The chronic complications of diabetic patients cause bone loss due to alterations in minerals and bone metabolism [41]. Macro and microvascular complications are seen in the diabetic patients. Coronary heart disease, cerebrovascular disease, and peripheral vascular disease comes under macro vascular complication where as effects on small vessels, arterioles, capillaries and venules comes under micro vascular complication [42]. Microvascular complication are more in adults and old age and rare in childhood and adolescence [43].

Prevention: Diabetes is found more even in younger children either due to genetic history of family or metabolic changes [44]. Proper dietary supplement with antioxidants may be useful for prevention of diabetes. The effective treatment for this disease is the ocular antioxidant potential therapy, which is harmless and improves the treatment without toxicity [45]. One can prevent diabetes by the intake of food, which contains the low percent of carbohydrates, proteins \& fat. Exercise and/or yogas are also one of the best methods for prevention of diabetes and also other metabolic syndromes. Avoiding high-carbohydrate food before sleep, taking high-protein breakfast and taking foods, which have low calories like fruits, vegetable, leans chicken, turkey, etc. will also control diabetes [46]. As discussed above zinc insulin prevents diabetes by taking twice daily instead of zinc [47]. The person who intake high supplements of $\omega-3$ fatty acids can be cured from the metabolic syndrome. It improves the defects in the insulin signaling and prevents the glucose homeostasis and results in the 
prevention in further development of type 2 diabetes [48]. Some of the pharmacological drugs are used for treating type 2 diabetes, compresses the progress of disease and helps in glycemic control due to loss of $\beta$-cell function [49]. Type 2 diabetes mellitus (T2DM) can also be prevented by oral anti-diabetic medications and maintaining correct lifestyle [50]. Impaired fasting glucose and glucose tolerance test in prediabetic stage have potential to reduce the risk of diabetes [51].

Obesity: Obesity caused due to fat consumption in the body not only from fat but also extra muscle, water content as well as increased bone weight [52]. Obesity not only associates with diabetes but also with cardiovascular diseases which result to oxidative stress [53]. Obesity mainly seen at central middle-age leads to type- 2 diabetes [54]. Latest research studies find out the parent-only (PO) treatment for childhood obesity, which shows effective results for weight loss in a child [55]. The obese people also have prevalence of obstructive sleep apnea (OSA), hypoxia and hypertension [56]. Obesity is also associates with malignant, metabolic and neurological disorders [57].

Cause: The main factors for obesity are:

1) Hereditary

2) Lifestyle

3) Psychological activities

Obesity is hereditary which is due to genetic cause, also due to changes in lifestyle i.e. laziness, and enjoyable lifestyle without exercise leads to obesity. Psychological problems like depression, sadness, stress, anxiety may also leads to obesity [58]. Increased level of also cortisol is also one of the causes for obesity [59].

Prevention: The effective way to prevent obesity is exercise, mainly walking and cycling. By drinking fat free drink and fruit juices without sugar, avoiding fast-foods and snacks every times, spicy foods in restaurants etc. can also prevent obesity [60]. New techniques are also developed to treat obesity which is a quick process. Some drugs are also released for weight loss.

Hormonal Imbalance: Disease like Adrenal imbalance, insulin Imbalance, thyroid imbalance, pituitary,sex hormones, imbalance toxicity of estrogen, etc.

Cause: The main hormones produced by ovary are estrogen, progesterone and testosterone due to imbalance in these hormones one can suffer from endometriosis, breast disease, menstrual irregularities and polycystic ovarian syndrome. Hormones like cortisol, aldosterone and androgens of adrenal glands have major role. Cortisol helps to maintain blood sugar and improve immune system, aldosterone balance salt and water in the body where as androgens enhances DHEA in men and women. Imbalances in these hormones in these gland leads to blood sugar irregularities, high androgen levels, problems in nervous and immune systems [61]. Imbalance in cytokine hormone causes Rheumatoid arthritis (RA), which is chronic metabolic disease [62].

Prevent: New technique hormone replacement therapy (HRT) is used to recover the disorders regarding hormonal imbalance. It is done in the patient with breast cancer, blood clots, liver disease and pregnancy to cure the disease. But there is some side effect during this therapy such as breast tenderness, headaches, dizziness, and blood clots etc [63].

Cardiovascular Disease: Cardiovascular diseases (CDV) are most commonly seen metabolic syndrome everywhere in the world. Nearly $80-90 \%$ of people have cardiovascular risk of the metabolic syndrome. The main causes for the risk factors are obesity, genetic disposition, insulin resistance and inflammation [64]. Cardio vascular risk increases in the patients who suffer from rheumatic diseases, which is bone metabolic syndrome [65]. The patients who suffer from diabetes mellitus have higher risk of cardiovascular disease which leads to morbidity and mortality [66]. The intake of heavy alcohol increases the risk of cardiovascular disease as well as hypertension [67].

Cause: The main factor for the cardiovascular disease is sedentary lifestyle, results to weight gain and further results to the cardiovascular risk. After intake of food it converts into glucose and enters into blood steam, unused glucose remains in blood stream. It is carried to liver and remains there as fat, which cause cardiovascular diseases. The main cause for cardiovascular risk in metabolic syndrome is high blood sugar levels, high triglycerides, high levels of bad cholesterol (HDL), low levels of good cholesterol (LDL) etc. [68].

Prevention: One can prevent the cardiovascular risks by avoiding

- Smoking; tobacco which contains nicotine leads high risk to heart and chance of causing cardiovascular diseases.

- Changes in diet: More fat intake and improper diet is also one of the ways to cause cardiovascular diseases. So we have to avoid fat foods and have to take proper diet.

- Exercise: At least of 30minutes exercise can keep the persons heart healthy.

- Alcohol: By avoiding alcohol intake one can decrease risk of cardiovascular.

- Weight: Overweight also increases the cardiovascular risk factor. So, we have to maintain correct weight according to height and age [69].

Drugs like vitamin C, B, E and folic acid are not recommended to decrease the cardiovascular risk factor [69].

\section{Conclusion}

In conclusion by change in the sedentary lifestyle and control on diet can prevent metabolic syndrome. Drugs and some vitamins like C, $\mathrm{B}$, and $\mathrm{E}$ are also some of the factors which alter the metabolic pathways. Having a control on all these factors prevents metabolic syndrome.

\section{References}

1. http://www.hormone.org/endocrine_system.cfm

2. Kaneko M, Suzuki H, Watanabe H, Oda E, Aizawa Y (2011) Metabolic Syndrome is a Poor Predictor of Incident Diabetes Compared with Hemoglobin A1c (Hba1c) in a General Japanese Population. J Diabetes Metab S: 2.

3. Vacek JL, Vanga SR, Good M, Lai SM, Lakkireddy D, et al. (2011) Vitamin D Deficiency and Supplementation and Relation to Cardiovascular Health. Am J Cardiol

4. Vestergaard P (2011) Diabetes and Bone. J Diabetes Metab S: 1

5. Uyemura K, Dhanani S, Yamaguchi DT, Song MK (2010) Metabolism and Toxicity of High Doses of Cyclo (his-pro) Plus Zinc in Healthy Human Subjects. J Drug Metabol Toxicol 1: 105. 5

6. Meeker JD, Rossano MG, Protas B, Diamond MP, Puscheck E, et al. (2009) Multiple metals predict prolactin and thyrotropin (TSH) levels in men.Environ Res 869-873.

7. Kuric L (2011) Molecular Biocoding of Insulin - Amino Acid Ser. J Bioengineer \& Biomedical Sci 1: 102.

8. Pavlov S, D Vasicek, J Kotwica, Sirotkin AV (2010) Involvement of Cell Cycle and Apoptosis-Related Protein p21 in Control of Secretory Activity of Porcine Ovarian Cells. Hormone Steroids J Sci 1: 102. 
9. http://en.wikipedia.org/wiki/Hormone_imbalance

10. Santos-Lozano A, Garatachea N (2011) Physical Activity Measurements Using Accelerometers and Pedometers in HIV-Infected People. J AIDS Clinic Res 2: 126

11. http://www.aidsmap.com/Metabolic-syndrome-as-common-in-HIV-negativeas-in-HIV-positive-individuals/page/1426286/

12. Gontran M, Jerome LT, Yvonne AM, Mavoungou-Poaty V, Elie M, et al. (2009) Effects of IM28 on HIV-1 and Metabolic Disorders-induced Highly Active Antiretroviral Therapy in Gabonese Patients. J Antivir Antiretrovir 1: 076-081.

13. Westmark CJ, Hervey CM, Berry-Kravisc EM, Maltera JS (2011) Effect of Anticoagulants on Amyloid ${ }^{\circledR}$-Protein Precursor and Amyloid Beta Levels in Plasma. J Alzheimers Dis 1: 101.

14. Aksoy P, Michael JB, Eduardo NC (2011) HMG-CoA Reductase Inhibitors Decrease Hyperglycemia on Animal Model of Systemic Inflammatory Response Syndrome (SIRS). J Anesthe Clinic Res 2: 165

15. Lakshminarasimhan N, Tagore S (2009) Damages in Metabolic Pathways: Computational Approaches. J Comput Sci Syst Biol 2: 208-215.

16. Morya VK, Dewaker V, Mecarty SD, Singh R (2010) In silico Analysis of Metabolic Pathways for Identification of Putative Drug Targets for Staphylococcus aureus. J Comput Sci Syst Biol 3: 062-069.

17. Ong YC, Su LH, Zaini A (2011) Reversal of Metabolic Dysfunction through Polyvalent Pharmacotherapy-augmented Lifestyle Intervention: Case Reports. J Diabetes Metab 2: 133

18. Chang Y (2011) A Central Role of PTP1B in Hyperinsulinemia-Enhanced IL-6 Signaling in Dedifferentiated Vascular Smooth Muscle Cells. J Diabetes Metab 2: 118.

19. http://en.wikipedia.org/wiki/Metabolic_syndrome

20. http://clinicaltrials.gov/ct2/show/NCT01014949

21. http://en.wikipedia.org/wiki/Nutrition

22. Belmokhtar F, Belmokhtar R, Charef M (2011) Risk Factors Associated With Type 2 Diabetes Mellitus in West Region of Algeria, Maghnia. J Diabetes Metab 2: 148 .

23. Chen D, Huang $H$, Xing $Y$, Liu $Y$, Xu Y, et al. (2011) A New Vanadium Complex Improves the Spatial Learning and Memory by Activation of Caveolin- MAPKCREB Pathway in Diabetic Mice. J Diabetes Metab 2: 114

24. Mansour AA, Wanoose HL, Odaa AH (2011) A Three Year Cohort Prospective Type 2 Diabetes Control Study in Basrah. J Diabetes Metab 2: 119.

25. Patra SR, Jahnavi G (2011) An Improvement in Compliance for Foot Care in Persons with Type 2 Diabetes with a Teaching Session. J Diabetes Metab 2: 130

26. Mungrue K, Roper LA, Chung T (2011) Assessment of Weight Loss in the Management of Patients with Type 2 Diabetes Mellitus in Primary Care in Trinidad. J Diabetes Metab 2: 120.

27. Shanker JH, Mahmood SE, Joshi MC, Shaifali I (2011) Obesity Indices amongs Diabetics in an Urban Population of Western Nepal. J Diabetes Metab 2: 134.

28. Kanazawa I (2011) Osteocalcin Possesses Hormonal Function Linking Bone to Glucose Metabolism. J Diabetes Metab 2: 105e.

29. Ali ZH (2011) Health and Knowledge Progress among Diabetic Patients after Implementation of a Nursing Care Program Based on Their Profile. J Diabet Metabol 2: 121.

30. Diabetes (2011) A.D.A.M. Medical Encyclopedia.

31. Alina S, Barbara R, Krzysztof G, Barbara G, Marek G, et al. (2011) Elevation of sE-Selectin Levels from 2-24 Months Following Gestational Diabetes is Associated with Early Cardiometabolic Risk in Non-Diabetic Women. J Diabetes Metab 2: 138.

32. Saini A, Devidayal, Verma S, Bhalla AK (2011) Comparative Efficacy of Once Daily Insulin Glargine with Twice Daily NPH Insulin in Children with Type 1 Diabetes. J Diabetes Metab 2: 124.

33. Uppu RM, Parinandi NL (2011) Insulin Sensitization and Resistance Interrelationship Revisited with a Quantitative Molecular Model Approach. J Diabetes Metab 2: $106 \mathrm{e}$.

34. Nichol A, ChandraSekar M (2011) Successful Management of Extremely
Insulin-Resistant Obese Diabetic Patient with Insulin Glargine, U-500 Regular Insulin and Pramlintide. J Diabetes Metab 2: 143

35. Taxitiemuer A, Yimamu Y, Mohemaiti P, Nuli R (2011) Serum Metabonomic Study of 2 Uyghur Probable MODY Families Based on 1H NMR. J Diabetes Metab 2: 122.

36. Hellwege JN, Hicks PJ, Palmer ND, Maggie CYN, Freedman BI, et al. (2011) Examination of Rare Variants in HNF4a in European Americans with Type 2 Diabetes. J Diabetes Metab 2: 145.

37. Lemos Costa TMR, Detsch JM, Pimazoni-Netto A, de Almeida ACR, SztalMazer S, et al. (2011) Glycemic Variability and Mean Weekly Glucose in the Evaluation and Treatment of Blood Glucose in Gestational Diabetes Mellitus: Evidence for Lower Neonatal Complications. J Diabetes Metab 2: 137

38. Kamoi K, Ohara N, Tomoo I, Shinozaki Y, Furukawa K (2011) Normal Response of Active GLP-1 Level like Substances to Test Meal in Non-Obese Type 2 Diabetic Japanese Patients with Complications and Receiving Treatments. $J$ Diabetes Metab 2: 147

39. Li Y (2011) Elevated Angiotensin II in Rat Nodose Ganglia Primes DiabetesBlunted Arterial Baroreflex Sensitivity: Involvement of NADPH OxidaseDerived Superoxide. J Diabetes Metab 2: 135.

40. Ragheb R, Medhat AM (2011) Mechanisms of Fatty Acid-Induced Insulin Resistance in Muscle and Liver. J Diabetes Metab 2: 127.

41. Abo-El-Asrar M, Farid SM, Maraghy MOE, Mohamedeen AK (2011) Serum Osteocalcin, Zinc Nutritive Status and Bone Turnover in Children and Adolescents with Type1 Diabetes Mellitus. J Diabetes Metab 2: 128.

42. http://www.aidsmap.com/Metabolic-syndrome-as-common-in-HIV-negativeas-in-HIV-positive-individuals/page/1426286/

43. El Asrar MA, Adly AAM, El Hadidi E, Gharib M (2011) Serum and Urinary Nitrites and Nitrates and Doppler Sonography in Detection of Early Diabetic Complications. J Diabetes Metab 2: 117

44. Madácsy L (2011) [Prediction and prevention of type 1 diabetes mellitus: initial results and recent prospects]. Orv Hetil 152: 1916-1921.

45. da Silva SB, Costa JP, Pintado ME, Ferreira DC, Sarmento B (2010) Antioxidants in the Prevention and Treatment of Diabetic Retinopathy - A Review. J Diabetes Metab 1: 111

46. http://diabetesinformationhub.com/DiabetesControl_DiabetesPrevention.php

47. Ramachandra S (2011) Do we need yet another Insulin? J Diabetes Metab 2 0e4

48. Tint D, Anghel M, Lupu DS, Fischer LM, Niculescu MD (2011) Low dose Flaxseed Oil Supplementation Alters the Fatty Acids Profile and the Progression of Metabolic Syndrome in Men without Adequate Medical Treatment. J Nutrition Disorder Ther S7: 001.

49. Ragheb R, Medhat AM (2011) Mechanisms of Fatty Acid-Induced Insulin Resistance in Muscle and Liver. J Diabetes Metab 2: 127.

50. Parvaresh E, Esteghamati A, Nakhjavani M, Aminorroaya A, Aboutorabi $\mathrm{R}$, et al. (2011) Biphasic Insulin Aspart 30 (BIAsp 30) is Safe and Improves Glycaemic Control in Insulin Naïve Patients with Type 2 Diabetes. J Diabetes Metab 2: 123.

51. Ramachandran A, Moses A, Snehalatha C, Shetty AS, Seeli AC et al (2011) Assessment of Sudomotor Function to Predict Future Abnormalities of Glucose Tolerance in at Risk Population. J Diabetes Metab 2: 125

52. Morbid obesity; Fat - obese (2011) A.D.A.M. Medical Encyclopedia

53. Lavoie M, Rabasa-Lhoret R, Ziai S, Lavoie J (2011) Blood Glutathione Peroxidase Activity in Relation with the Risk of Cardiovascular Diseases in Obese Women. J Diabetes Metab 2:136.

54. Hansen BC, Shamekh R, Hansson O, Almgren P, Budagov T, et al. (2011) The Rhesus Monkey: A Nonhuman Primate Model For T2DM- Associated Gene Screening. J Diabetes Metab 2: 150.

55. http://www.nature.com/oby/journal/vaop/ncurrent/abs/oby2010238a.html

56. Gayes JM (2011) Obesity, Obstructive Sleep Apnea and the "HELP" Position. J Anesthe Clinic Res 2:115.

57. Majithia R, Koch TR (2011) Our Obesity Crisis Requires The Development of New, Widely Available Options:Can Yoga Function In A Major Role? J Yoga Phys Therapy 1: e102.

58. http://www.strengthforcaring.com/conditions/obesity/types/ 
59. Tõnisson M, Tillmann V, Kuudeberg A, Väli M (2011) Effect of CBT on Depressive Symptoms in Methadone Maintenance Patients Undergoing Treatment for Hepatitis C. J Addict Res Ther 2: 111.

60. http://www.a1articles.com/prevention-of-obesity-1018133.html

61. http://www.zrtlab.com/hormone-testing/symptoms-of-hormone-imbalance.html

62. Heruth DP, Zhang LQ, Ye SQ (2011) Nicotinamide Phosphoribosyltransferase in Rheumatoid Arthritis. J Bioanal Biomed 3: 076-080.

63. 'http://en.wikipedia.org/wiki/Hormone_imbalance

64. Qiao Q, Gao W, Zhang L, Nyamdorj R, Tuomilehto J (2007) Metabolic syndrome and cardiovascular disease. Ann Clin Biochem 44: 232-263.
65. Martocchia A, Toussan L, Stefanelli M, Falaschi GM, Comite F, et al. (2011) Association of Severity of Osteoarthritis and Carotid Atherosclerosis in Patients with Metabolic Syndrome. Rheumatology 1: 105.

66. Li YW, Aronow WS (2011) Diabetes Mellitus and Cardiovascular Disease. J Clinic Experiment Cardiol 2: 114

67. Li H, Wang G, Wang A, Tong W, Zhang Y (2011) Alcohol Consumption and Risk of Type 2 Diabetes in Mongolian Population, Inner Mongolia, China. J Diabet Metabol 2: 116.

68. http://metabolic-syndrome.insulitelabs.com/

69. http://www.who.int/cardiovascular_diseases/guidelines/Full\%20text.pdf 